\title{
Functionalization of Carbon Nanofibres Obtained by Floating Catalyst Method
}

\author{
Adolfo Fernández, ${ }^{1}$ Pavel Peretyagin, ${ }^{2}$ Washington Solís, ${ }^{2}$ \\ Ramón Torrecillas, ${ }^{1,2}$ and Amparo Borrell ${ }^{1,3}$ \\ ${ }^{1}$ Centro de Investigación en Nanomateriales y Nanotecnología (CINN) (CSIC, Universidad de Oviedo, Principado de Asturias), \\ Avenida de la Vega 4-6, 33940 El Entrego, Spain \\ ${ }^{2}$ Moscow State University of Technology (STANKIN), Vadkovskij per. 1, Moscow, Moscow Oblast, Russia \\ ${ }^{3}$ Instituto de Tecnología de Materiales (ITM), Universitat Politècnica de València, Camino de Vera, s/n, 46022 Valencia, Spain
}

Correspondence should be addressed to Adolfo Fernández; a.fernandez@cinn.es

Received 27 January 2015; Accepted 13 March 2015

Academic Editor: Nay Ming Huang

Copyright (C) 2015 Adolfo Fernández et al. This is an open access article distributed under the Creative Commons Attribution License, which permits unrestricted use, distribution, and reproduction in any medium, provided the original work is properly cited.

\begin{abstract}
The excellent physicochemical and electrical properties of carbon nanofibres (CNF) combined with the possibility of being produced at industrial scale at reasonable costs have promoted the interest in their use in very diverse areas. However, there are still some drawbacks that must be solved in order to optimize their set of properties such as the presence of impurities or the imperfections in the crystalline structure. In this work, different modification treatments of CNFs produced by the floating catalyst method have been studied. Three types of modification processes have been explored that can be grouped as mechanical, thermal, and chemical functionalization processes. Mechanical processing has allowed solving the agglomeration problem related to CNFs produced by floating catalyst method and the resulting modified product ensures the secure handling of carbon nanofibres. Thermal and chemical treatments lead to purer and more crystalline products by removing catalyst impurities and amorphous carbon. Functionalization processes explored in this work open the possibility of customized posttreatment of carbon nanofibres according to the desired requirements.
\end{abstract}

\section{Introduction}

The study of carbon nanomaterials such as carbon nanotubes, carbon nanofibres, and graphene has emerged during the last years due to their excellent physicochemical properties and their huge potential applications [1-4]. Carbon nanofilaments are produced since more than two decades by using different catalysers with hydrocarbon sources in gaseous phase [5-7]. However, the interest in this type of materials clearly increased after the discovery of multiwall nanotubes (MWNT) by Iijima [8] in 1991, single wall nanotubes (SWNT) by Iijima and Ichihashi [9] and Bethune et al. [10] in 1993, and more recently graphene [11]. Nowadays, the research on carbon nanomaterials is one of the most active research topics in the nanoscience and nanotechnology fields.

Carbon nanofibres (CNFs) are a very attractive material from the economic point of view. CNFs combine very interesting mechanical and electrical properties with reduced production costs in comparison with carbon nanotubes (CNTs), what could facilitate their incorporation to different mass production industrial sectors such as reinforcing phases in thermostable or thermoplastic polymers, supercapacitors, ion-lithium batteries, gas storage, catalyzer supports, and electroconductive ceramics. CNFs are dimensionally similar to CNTs but they differ in the structure. Thus, CNFs show nonconcentric graphene planes and they are classified in different types according to their structure [12], such as "platelet," "fishbone," "ribbon," or "stacked cup."

From a general point of view, CNFs growth in gaseous phase can be described as a solid material with length: diameter ratio higher than $10: 1$, a diameter $<100 \mathrm{~nm}$, and a carbon content $>90 \%$. The synthesis takes place by the catalytic decomposition of a carbon source (hydrocarbons or CO) at a temperature ranging from 500 to $1200^{\circ} \mathrm{C}$ and using a transition metal such as $\mathrm{Fe}, \mathrm{Co}, \mathrm{Ni}$, or $\mathrm{Cu}$ as catalyzer. 
Among the different methods of production in gaseous phase, the floating catalyst method is especially interesting as it allows the continuous production of CNFs enabling the industrial use of this product. In this work, they are used CNFs produced by floating catalyst method. Nickel was used as catalyzer [13]. Briefly, the solution of nickel catalyzer is injected in the reactor and a sulfur source is added [14] while from the upper part of the reactor it incorporated natural gas as carbon source and hydrogen gas as carrier keeping a reductive atmosphere during the process. Synthesis temperature is controlled around $1100^{\circ} \mathrm{C}$. The importance of the sulfur role during CNFs synthesis has been previously described [15-18], which must be added independently from the catalyzer used.

Despite the economic advantages related to CNFs production, their lower properties in comparison with CNTs are limiting their incorporation as a mass product in the market. The main features that must be improved in the case of CNFs are three, chemical purity, structural order, and surface properties. The objective of this work is to functionalize the as synthesized CNFs by different treatments. The purpose is to enhance their final properties in order to increase their interest as advanced material. In particular, different mechanical, chemical, and thermal treatments were studied and the evolution of the CNFs properties was evaluated in comparison with raw CNFs.

\section{Materials and Methods}

2.1. Starting Materials. In Table 1, the properties of the starting carbon nanofibres used in this work are described.

2.2. Description of Posttreatment Procedures. Functionalization processes studied in this work can be divided in three subcategories, mechanical, thermal, and chemical posttreatments.

2.2.1. Mechanical Modification. Mechanical treatments were carried out with a high energy attrition mill (Union Process, USA). The attrition mill has a central axis equipped with perpendicular ceramic bars that is placed into a container filled with alumina media of $2 \mathrm{~mm}$ diameter and the material (CNFs) using anhydrous $99.97 \%$ ethanol as solvent. Then, $350 \mathrm{rpm}$ rotation speed was applied for 1 hour. After this time, rotation speed was reduced to $50 \mathrm{rpm}$ and the slurry was extracted through the exit at the bottom of the container. This slurry was firstly dried at $60^{\circ} \mathrm{C}$ and the resulting cake was finally dried at $120^{\circ} \mathrm{C}$. Finally, the dried powder was passed through a $63 \mu \mathrm{m}$ sieve.

2.2.2. Thermal Treatments. Different thermal treatments were used in order to study their influence on the purity and the crystallinity order of CNFs. Thermal treatments cover from relatively low temperature carbonization process to high temperature graphitization tests.

Carbonization treatments were carried out in a tubular furnace (Agni, Germany), under continuous flow of $500 \mathrm{~mL} / \mathrm{min}$ of high purity nitrogen (Alphagaz, Air Liquide)
TABle 1: Properties of starting nanofibers.

\begin{tabular}{lc}
\hline Nanofibre average diameter & $20-80 \mathrm{~nm}$ \\
Nanofibre average length & $>30 \mu \mathrm{m}$ \\
Density & $>1.97 \mathrm{~g} / \mathrm{cm}^{3}$ \\
Specific surface & $150-200 \mathrm{~m}^{2} / \mathrm{g}$ \\
Graphitization degree & $\sim 70 \%$ \\
Electrical resistivity & $10^{-2} \Omega \cdot \mathrm{cm}$ \\
Metallic particles content $(\mathrm{Ni})$ & $6-8 \mathrm{wt} \%$ \\
\hline
\end{tabular}

at temperature range $1100-1500^{\circ} \mathrm{C}$ for two hours. $2 \mathrm{~g}$ of $\mathrm{CNFs}$ introduced in a ceramic crucible was used in each test.

On the other hand, graphitization test was carried out with $1 \mathrm{~g}$ of CNFs previously carbonized that was heated in a graphite crucible up to $2700^{\circ} \mathrm{C}$ in a graphite furnace, under argon flow, at a heating rate of $10^{\circ} \mathrm{C} / \mathrm{min}$. Sample was kept at this temperature for $1 \mathrm{~h}$.

2.2.3. Modification with Chemical Solvents. Different chemical reagents were used for exploring the surface modification of CNFs and the efficiency of removing impurities. $\mathrm{HCl}$, $\mathrm{HNO}_{3}, \mathrm{H}_{2} \mathrm{O}_{2}, \mathrm{H}_{3} \mathrm{PO}_{4}$, and $\mathrm{H}_{2} \mathrm{SO}_{4}$ (Sigma-Aldrich) were used as chemical reagents. In a typical experiment, CNFs are mixed with the chemical solvent in 1:100 weight ratios and the mixture is heated under reflux at $80^{\circ} \mathrm{C}$ for 1 hour. Then, the slurry is filtered and washed with distilled water until no variation of the water $\mathrm{pH}$ is observed. Then, the solid is dried at $120^{\circ} \mathrm{C}$ for 48 hours.

\subsection{Characterization Techniques}

2.3.1. Chemical Analysis. Carbon, hydrogen, nitrogen, and sulfur content was determined by combustion of $1 \mathrm{mg}$ of sample in a LECO-CHNS-932 apparatus. Oxygen content was determined by direct measuring using a graphite furnace LECO-VTF-900 coupled with the apparatus previously described.

Nickel content was determined by atomic absorption spectroscopy (apparatus AA-6300 Shimadzu) with background correction with deuterium lamp (BGD-D2). The procedure included the wet extraction of nickel with microwaves.

2.3.2. Raman Spectroscopy. Raman spectroscopy was carried out with a Raman modular XY apparatus (DILOR), with CCD4 detector using a $532 \mathrm{~nm}$ excitation source (laser spectra-physics 265). The power was $20 \mathrm{~mW}$ and slit width was $200 \mu \mathrm{m}$. Spectra were deconvoluted by Lorentzian/Gaussian adjustment.

2.3.3. Scanning Electron Microscopy. Micrographs of synthesized and postmilled carbon nanofibres were obtained with scanning electron microscopy (SEM, Zeiss DSM 950).

2.3.4. Differential Thermal Analysis and Thermogravimetry. DTA and TG analyses were carried out using TA INSTRUMENTS Model SDT 2960 and TGA/DSC1 METTLER TOLEDO GC 200 equipment, respectively. These apparatus 


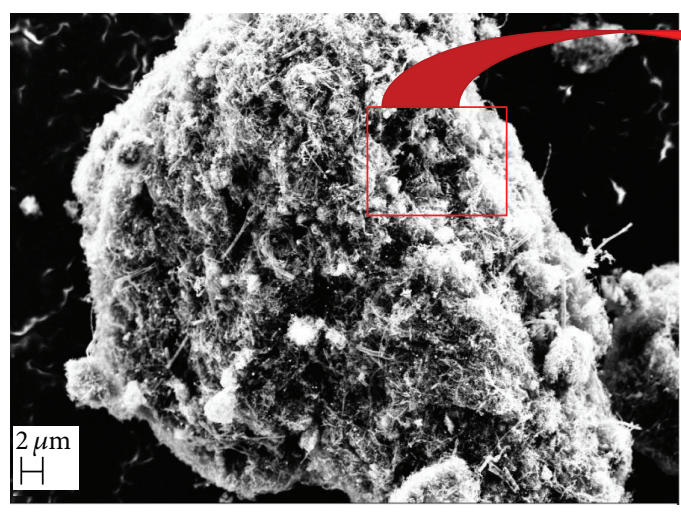

(a)

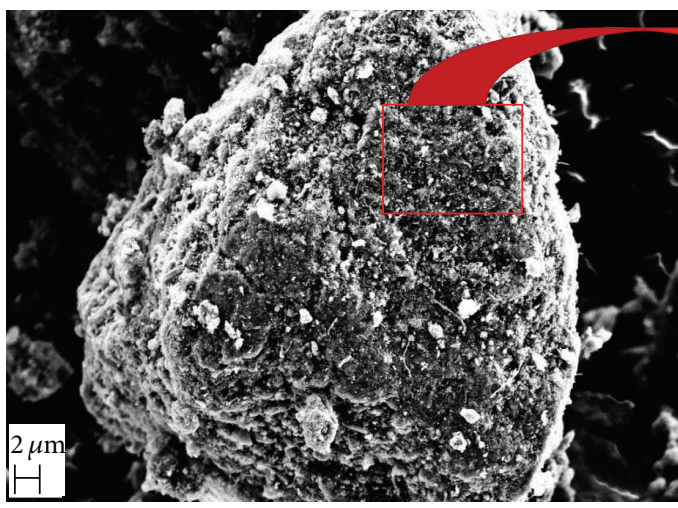

(c)

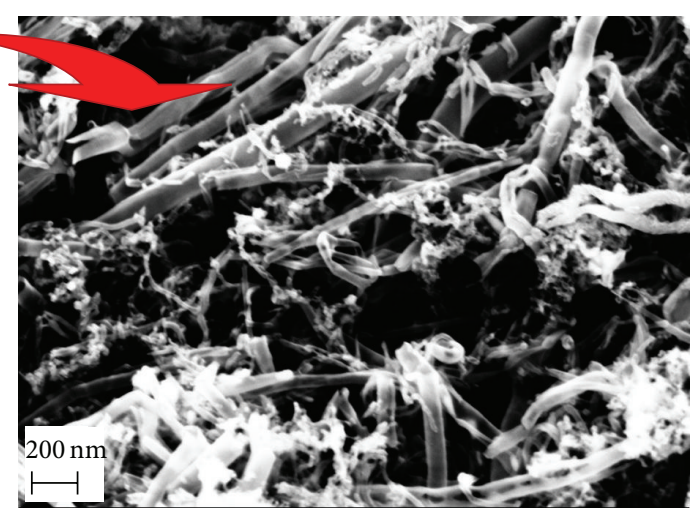

(b)

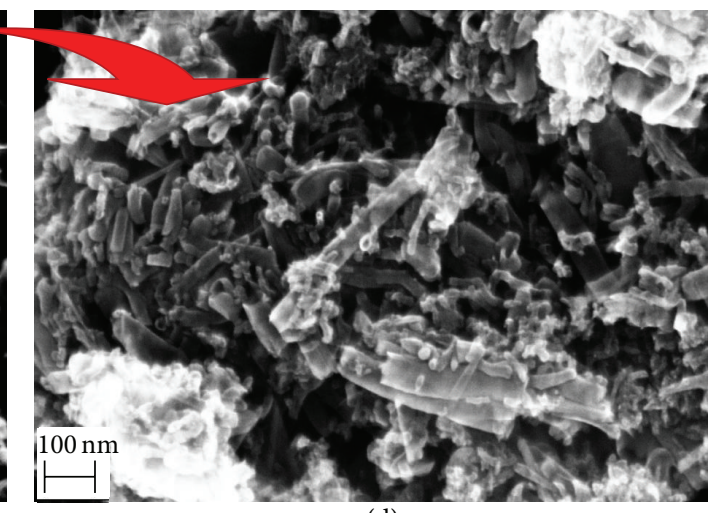

(d)

FIGURE 1: SEM micrographs of original ((a) and (b)) and mechanically milled ((c) and (d)) CNFs.

analyzed both phenomena simultaneously either under inert atmosphere or air. They used Pt crucible for all the tests.

\section{Results and Discussion}

3.1. Modification by Attrition Milling. The CNFs used in this work were produced by the floating catalyst method; that is, a catalyst is injected at high temperature in presence of a hydrocarbon source. As consequence, the product is obtained while the current is passing the chamber in form of balls where the CNFs are intertwined [19]. A first negative consequence of this result is the difficulty for dispersing the CNFs in polymeric or ceramic matrices. Secondly, the low density of the CNFs balls due to the air occluded inside enhances the liberation to the environment during handling increasing the security risks that could be even more important in an industrial scale use. Then, the first posttreatment studied was the mechanical milling of CNFs balls by attrition milling in order to condition the raw material for more secure handling and in order to get a more easily dispersible product.

In Figure 1 the micrographs obtained by SEM corresponding to starting and milled CNFs are shown, respectively. In the low magnification micrographs ((a) and (c)) it is observed that the average sizes of microgranules are similar. Granule of starting CNFs corresponds to produced nanofibres while the granule size of milled CNFs can be adjusted depending on the sieve used. Then, the use of $63 \mu \mathrm{m}$ sieve allows obtaining granule with similar average size as the original CNFs. Nevertheless, the comparison of high magnification micrographs ((b) and (d)) shows how the granules obtained after milling are significantly more compacted. They are formed by shorter nanofibres efficiently packed while the original granules are formed by larger and knotted nanofibres. This result has been quantified by measuring the tap density following the ISO 23145-1:2007 standard. Tap density of CNFs increased from 0.08 to $0.41 \mathrm{~g} / \mathrm{cm}^{3}$ after the attrition milling process, that is, five times higher as consequence of the efficient compaction as it was observed by SEM. It has been also proved that milled CNFs handling is similar to conventional micrometer size powders. This result is very promising from the security point of view.

In Table 2, the results of the chemical analysis of raw CNFs and the different products obtained after the functionalization posttreatments are shown. In the case of milled CNFs, as it could be expected, chemical composition is very similar to raw CNFs. A slightly simultaneous decrease of $\mathrm{C}$ content and increase of $\mathrm{H}$ and $\mathrm{O}$ are only observed. This result is due to the presence of low amounts of ethanol in milled CNFs. It has been quantified by TG/DTA that around $3 \mathrm{wt} \%$ of ethanol is absorbed in milled CNFs.

3.2. Purification by Thermal Treatments. Production of CNFs by the floating catalyst procedure is a very efficient method from the point of view of industrial scaling-up but the short times of contact among the catalyst particles and the carbon sources lead to incomplete transformation of hydrocarbon 


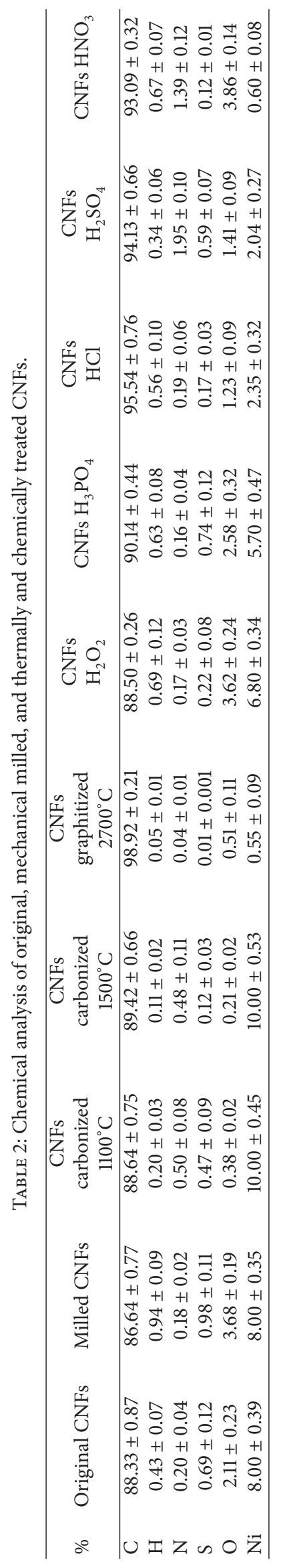


into graphitic products. Thus, from the chemical analysis of produced CNFs it can be observed that the $\mathrm{C}$ content is $88 \mathrm{wt} \%$ ( $~ 95 \mathrm{wt} \%$ excluding $\mathrm{Ni}$ ) including amorphous carbon. Then, this section explores the possibility of increasing CNFs purity by applying different thermal treatments from carbonization processes for removing volatile compounds to high temperature graphitization treatments for improving the crystallinity degree of CNFs.

It is known that thermal treatments at temperatures higher than the melting point of condensed product are efficient for increasing the purity of the carbon material [20-22]. Thus, two carbonization treatments were studied, the final temperatures being 1100 and $1500^{\circ} \mathrm{C}$, respectively, and the stay at the maximum temperature $2 \mathrm{~h}$. In Table 2 the results from the chemical analysis can be seen. It is observed how the sulfur content is reduced to $85 \%$ after heating at $1500^{\circ} \mathrm{C}$. It is expected that residual sulfur is forming nickel sulfide in the catalyst particles [19]. The reduction in oxygen content is also noticeable, even for the lower temperature treatment $\left(1100^{\circ} \mathrm{C}\right)$. These results together with the progressive reduction in hydrogen content are proving the removal of volatile compounds enriched in $\mathrm{O}, \mathrm{S}$, and $\mathrm{H}$ and, therefore, an increase in the $\mathrm{C}$ content. The increase in the relative content of $\mathrm{Ni}$ due to the removal of the volatile compounds hinders the increase in $\mathrm{C}$ content in CNFs. Thus, if the relative content in $\mathrm{C}$ is recalculated excluding the $\mathrm{Ni}$ content, it is observed how the C content is increased from $95 \%$ for the original CNFs to $98 \%$ and $99 \%$ when the sample is carbonized at 1100 and $1500^{\circ} \mathrm{C}$, respectively.

Removal of volatile compounds can also modify the thermal stability of CNFs. The resistance to burn of original and carbonized CNFs by TG/DTA analysis under air atmosphere was studied. The resulting curves are shown in Figure 2. A significant delay in the starting burning temperature in the case of carbonized CNFs is observed. Whereas original CNFs burn from $510^{\circ} \mathrm{C}$, CNFs carbonized at 1100 and $1500^{\circ} \mathrm{C}$ start at 570 and $600^{\circ} \mathrm{C}$, respectively. Then, stability against oxidation has been significantly improved $\left(\sim 100^{\circ} \mathrm{C}\right)$ which is especially interesting for those applications in which the final component can be submitted to high temperatures such as ceramic-CNFs composites.

Following the strategy of purification by thermal treatments, the effect of a graphitization treatment at $2700^{\circ} \mathrm{C}$ was also studied. Graphitization of CNFs has been previously studied in order to evaluate the morphological changes in their graphitic structure [23-25]. From the data shown in Table 2, the effectivity of the graphitization treatment from the purification point of view can be clearly observed. Thus, the $\mathrm{C}$ content is $99 \%$ and only residual $\mathrm{O}$ and $\mathrm{Ni}$ contents are detected. In concordance with the results previously discussed for carbonized CNFs, the resistance to oxidation is further improved in the case of graphitized CNFs and the starting burning temperature is delayed until $650^{\circ} \mathrm{C}$.

The second objective of the thermal treatments is to improve the CNFs crystallographic degree. This effect was studied by Raman spectroscopy. Figure 3 shows the Raman spectra corresponding to original CNFs and CNFs thermaltreated at 1100,1500 , and $2700^{\circ} \mathrm{C}$. First order spectra (1200$1700 \mathrm{~cm}^{-1}$ ) only show two peaks, $\mathrm{G} \sim 1580 \mathrm{~cm}^{-1}$ related with

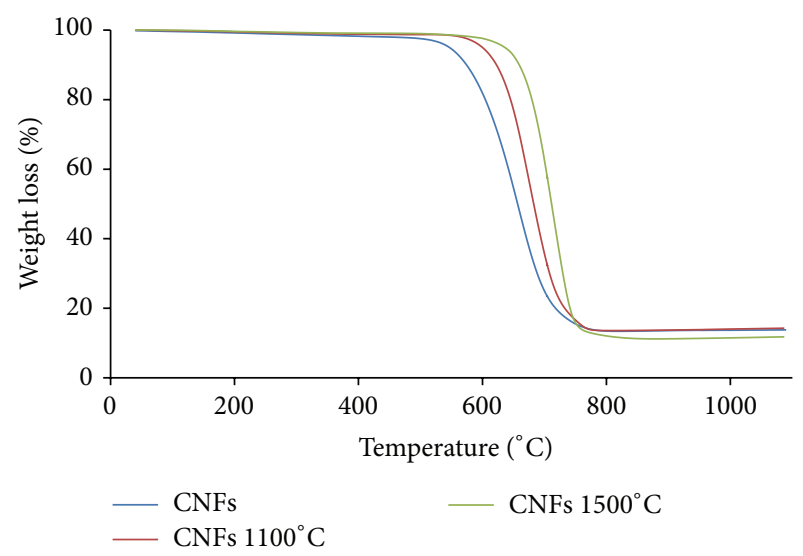

FIgURE 2: TG curves under air atmosphere of original and carbonized CNFs.

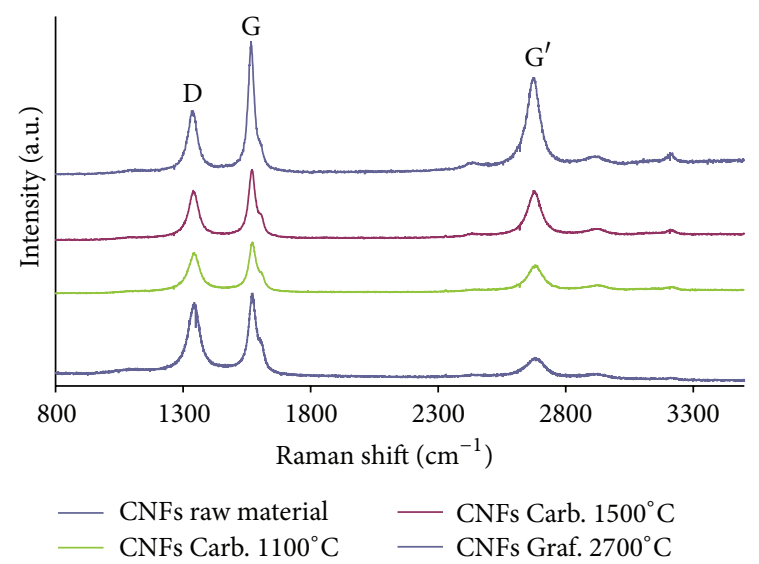

Figure 3: Raman spectra of original and thermally treated CNFs.

graphitic material and $\mathrm{D} \sim 1350 \mathrm{~cm}^{-1}$ related with imperfections in the crystalline structure. In the second order spectra (2500-2900 $\left.\mathrm{cm}^{-1}\right), \mathrm{G}^{\prime}$ appears as the only well-defined band $\sim 2700 \mathrm{~cm}^{-1}$. These bands allow comparing the crystalline order of carbon materials [26]. A significant increase in the relative intensity of $G$ and $\mathrm{G}^{\prime}$ bands is observed accompanied with a decrease in the band width when the temperature of the thermal treatment is increased, especially in the case of graphitization process at $2700^{\circ} \mathrm{C}$. Table 3 shows the relative intensity of D band and the position of $\mathrm{G}$ band in order to compare the results quantitatively. The decrease of relative $\mathrm{D}$ band intensity when the CNFs are thermal-treated is observed, especially in the case of graphitized nanofibres as well as the displacement of $\mathrm{G}$ band to higher wavenumber. Both results are related with an increase in the structural order.

3.3. Purification by Chemical Treatments. Finally, the chemical purification of synthesized CNFs has been studied. CNFs contain Ni nanoparticles from the synthesis and removal of these metal impurities can be interesting for specific applications. Moreover, the surface functionalization with chemical groups can enhance the dispersion of CNFs in ceramic 
TABLE 3: Parameters calculated from first order Raman spectra for original and thermally treated CNFs.

\begin{tabular}{lcc}
\hline Material & $v_{G}$ & $I_{D} / I_{T}$ \\
\hline CNFs & 1572.9 & 55 \\
CNFs $1100^{\circ} \mathrm{C}$ & 1573.2 & 49 \\
CNFs $1500^{\circ} \mathrm{C}$ & 1575.3 & 50 \\
$\mathrm{CNFs} 2700^{\circ} \mathrm{C}$ & 1582.2 & 35 \\
\hline
\end{tabular}

$v_{G}$ corresponds to $G$ band position $\left(\mathrm{cm}^{-1} \pm 1\right)$ and $I_{D} / I_{T}$ relative intensity ratio $I_{T}=\left(I_{D}+I_{G}\right)$.

and polymeric matrices which is a great challenge. Taking into account both objectives simultaneously, treatment with chemical reagents appears as a very promising method. Thus, chloride acid is recognized as a very suitable reagent for dissolving metals while nitric and sulfuric acids have more oxidant power $[27,28]$.

In this work, the effect of using five different chemical reagents has been studied, $\mathrm{HCl}, \mathrm{HNO}_{3}, \mathrm{H}_{2} \mathrm{O}_{2}, \mathrm{H}_{3} \mathrm{PO}_{4}$, and $\mathrm{H}_{2} \mathrm{SO}_{4}$. Table 2 shows the results of the chemical analysis of resulting CNFs. Concerning $\mathrm{Ni}$ removal, the most efficient chemical reagent was $\mathrm{HNO}_{3}$ that reduced the $\mathrm{Ni}$ content to $<1 \%$. The high oxidant power of nitric acid which can facilitate the partial oxidation of CNFs [29] allowing the extraction of Ni occluded inside the CNFs is known. On the other side, $\mathrm{H}_{2} \mathrm{O}_{2}$ leads to the lowest Ni removal rate. $\mathrm{H}_{2} \mathrm{O}_{2}$ has partially oxidized the nanofibre as it can be concluded from the oxygen content similar to the corresponding $\mathrm{HNO}_{3}$ treated CNFs but without effective $\mathrm{Ni}$ dissolution. Concerning $\mathrm{HCl}$, $\mathrm{H}_{2} \mathrm{SO}_{4}$, and $\mathrm{H}_{3} \mathrm{PO}_{4}$ treatments, they lead to partial reduction of $\mathrm{Ni}$ content. The result obtained after $\mathrm{H}_{3} \mathrm{PO}_{4}$ treatment is remarkable in which a slight reduction of Ni content is observed but $\mathrm{S}$ content remains similar to original CNFs. Catalyst particles are formed by Ni/Ni sulfide [19]. Then, it can be deduced that $\mathrm{H}_{3} \mathrm{PO}_{4}$ treatment dissolves selectively the metallic particles. This result has been corroborated by XRD where nickel sulfide (NiS) peaks are easily detected.

The resistance to oxidation of resulting CNFs after chemical modification has been studied. Burning profile is similar but significant differences can be observed from a more detailed analysis of the data shown in Table 4, where temperatures of initial weight loss, final weight loss, and maximum weight loosing rate are described. Starting burning temperature slightly decreases after chemical modification especially in the case of $\mathrm{HNO}_{3}$ treatment. The presence of more oxygenated groups facilitates the starting of material removal. However, the final temperature is similar for all the samples with a maximum difference of $<20^{\circ} \mathrm{C}$. Despite the lower temperature to CNFs burning after chemical treatments, the maximum rate of weight losses is delayed and, therefore, the average stability of the nanofibres is increased. During the chemical treatments, impurities and low crystallinity components adhered to CNFs are removed. Although the partial oxidation of carbon nanofibres allows an early starting of material removal, after the elimination of the generated oxygenated groups, the remaining material is more stable as it is mainly formed by high crystallinity material.
TABLE 4: TG analysis under air atmosphere of original and chemically treated CNFs.

\begin{tabular}{lllr}
\hline Sample & $T_{i}{ }^{\mathrm{a}}$ & $T_{f}{ }^{\mathrm{b}}$ & $T_{\max }{ }^{\mathrm{c}}$ \\
\hline $\mathrm{CNFs}$ & 480 & 710 & 550 \\
$\mathrm{CNFs} \mathrm{H}_{2} \mathrm{O}_{2}$ & 480 & 715 & 578 \\
$\mathrm{CNFs} \mathrm{H}_{3} \mathrm{PO}_{4}$ & 470 & 704 & 580 \\
$\mathrm{CNFs} \mathrm{HCl}_{\mathrm{CNFs} \mathrm{H}} \mathrm{SO}_{4}$ & 473 & 700 & 606 \\
$\mathrm{CNFs} \mathrm{HNO}_{3}$ & 473 & 700 & 603 \\
\hline
\end{tabular}

${ }^{\text {a }}$ Starting temperature of weight loss $\left({ }^{\circ} \mathrm{C}\right)$.

${ }^{\mathrm{b}}$ Final temperature of weight loss $\left({ }^{\circ} \mathrm{C}\right)$.

${ }^{c}$ Temperature for maximum rate of weight loss.

\section{Conclusions}

Mechanical, thermal, and chemical functionalizations of carbon nanofibres are efficient treatments for improving different properties of nanofibres obtained by floating catalyst method.

Original CNFs are knotted avoiding their efficient dispersion and increasing the health risk during handling due to their extremely low tap density. Mechanical milling allows partial nanofibre breaking eliminating knots and leading to compact and easy dispersible granules. Health risk during handling is minimized and the dispersion of milled product is noticeably improved.

Evolution of crystalline order of CNFs with temperature used during thermal treatments was studied by Raman spectroscopy. The efficiency of the treatment is proportional to the final temperature. Partial removal of amorphous carbon can be obtained at relatively low temperature carbonization treatment $\left(1100^{\circ} \mathrm{C}\right)$ while completely pure and high crystalline product is obtained after graphitization process.

Finally, chemical treatment with different reagents has been explored. Removal of catalyst impurities can be completed by treatment with $\mathrm{HNO}_{3}$ at low temperature $\left(80^{\circ} \mathrm{C}\right)$. Resistance to oxidation of resulting carbon nanofibres is improved thanks to the removal of impurities and amorphous carbon.

The results obtained in this work open the possibility of designing specific posttreatments of CNFs depending on the desired final properties that would be defined by the final application requirements.

\section{Conflict of Interests}

The authors declare that there is no conflict of interests regarding the publication of this paper.

\section{Acknowledgments}

Authors would like to thank Grupo Antolín Ingeniería for providing the starting carbon nanofibre. Authors would also like to thank The Ministry of Education of the Russian Federation for supporting this work by contract no. 14.577.21.0089, unique identifier of contract RFMEFI57714X0089. 


\section{References}

[1] M. S. Dresselhaus, G. Dresselhaus, and P. Avouris, Carbon Nanotubes: Synthesis, Structure, Properties and Applications, Springer, Berlin, Germany, 2001.

[2] E. V. Basiuk and V. A. Basiuk, "Green chemistry of carbon nanomaterials," Journal of Nanoscience and Nanotechnology, vol. 14, no. 1, pp. 644-672, 2014.

[3] K. Scida, P. W. Stege, G. Haby, G. A. Messina, and C. D. García, "Recent applications of carbon-based nanomaterials in analytical chemistry: critical review," Analytica Chimica Acta, vol. 691, no. 1-2, pp. 6-17, 2011.

[4] L. Dai, D. W. Chang, J.-B. Baek, and W. Lu, "Carbon nanomaterials for advanced energy conversion and storage," Small, vol. 8, no. 8, pp. 1130-1166, 2012.

[5] M. S. Dresselhaus, G. Dresselhaus, K. Sugihara, L. Spain, and H. A. Goldberg, Graphite Fibres and Filaments, Springer, Berlin, Germany, 1988.

[6] J. L. Figueiredo, C. A. Bernardo, R. T. K. Baker, and K. J. Hüttinger, Carbon Fibers Filaments and Composites, Kluwer Academic, Dordrecht, Netherlands, 1990.

[7] N. Latorre, T. Ubieto, C. Royo et al., "Materiales nanocarbonosos: nanotubos y nanofibras de carbono: aspectos básicos y métodos de producción," Ingeniería Química, vol. 36, no. 417, pp. 200-208, 2004.

[8] S. Iijima, "Helical microtubules of graphitic carbon," Nature, vol. 354, no. 6348, pp. 56-58, 1991.

[9] S. Iijima and T. Ichihashi, "Single-shell carbon nanotubes of 1nm diameter," Nature, vol. 363, no. 6430, pp. 603-605, 1993.

[10] D. S. Bethune, C. H. Kiang, M. S. De Vries et al., "Cobaltcatalysed growth of carbon nanotubes with single-atomic-layer walls," Nature, vol. 363, no. 6430, pp. 605-607, 1993.

[11] A. K. Geim and K. S. Novoselov, "The rise of graphene," Nature, vol. 6, no. 3, pp. 183-191, 2007.

[12] I. Martin-Gullon, J. Vera, J. A. Conesa, J. L. González, and C. Merino, "Differences between carbon nanofibers produced using $\mathrm{Fe}$ and Ni catalysts in a floating catalyst reactor," Carbon, vol. 44 , no. 8, pp. 1572-1580, 2006.

[13] C. Merino and P. Soto, "Furnace for the manufacture of carbon fibres, procedure for obtaining using said furnace and the fibre thus obtained," European Patent Application 04381014, 2004.

[14] G. G. Tibbetts, C. A. Bernardo, D. W. Gorkiewicz, and R. L. Alig, "Role of sulfur in the production of carbon fibers in the vapor phase," Carbon, vol. 32, pp. 569-576, 1994.

[15] S. Collins, R. Brydson, and B. Rand, "Structural analysis of carbon nanofibres grown by the floating catalyst method," Carbon, vol. 40, no. 7, pp. 1089-1100, 2002.

[16] L. Ci, Y. Li, B. Wei, J. Liang, C. Xu, and D. Wu, "Preparation of carbon nanofibers by the floating catalyst method," Carbon, vol. 38, no. 14, pp. 1933-1937, 2000.

[17] L. Ci, H. Zhu, B. Wei, J. Liang, C. Xu, and D. Wu, "Phosphorusa new element for promoting growth of carbon filaments by the floating catalyst method," Carbon, vol. 37, no. 10, pp. 1652-1654, 1999.

[18] C. Singh, T. Quested, C. B. Boothroyd et al., "Synthesis and characterization of carbon nanofibers produced by the floating catalyst method," The Journal of Physical Chemistry B, vol. 106, no. 42, pp. 10915-10922, 2002.

[19] J. V. Agulló, Nanofilamentos de carbono, sus tratamientos superficiales y su aplicación en materiales compuestos de matriz polimérica [Doctoral thesis], University of Alicante, Alicante, Spain, 2008.
[20] Y. A. Kim, T. Matusita, T. Hayashi, M. Endo, and M. S. Dresselhaus, "Topological changes of vapor grown carbon fibers during heat treatment," Carbon, vol. 39, no. 11, pp. 1747-1752, 2001.

[21] S. Lim, S. H. Yoon, I. Mochida, and J. H. Chi, "Surface modification of carbon nanofiber with high degree of graphitization," Journal of Physical Chemistry B, vol. 108, no. 5, pp. 1533-1536, 2004.

[22] G. G. Tibbetts, D. G. Glasgow, C. Kwag, J. Y. Howe, and M. L. Lake, How Structural Changes Induced by Heat-Treatment of Carbon Nanofibers Can Lead to Improved Composite Properties, Carbon, Providence, RI, USA, 2004.

[23] J. Chen, J. Y. Shan, T. Tsukada et al., "The structural evolution of thin multi-walled carbon nanotubes during isothermal annealing," Carbon, vol. 45, no. 2, pp. 274-280, 2007.

[24] M. Endo, Y. A. Kim, T. Hayashi et al., "Microstructural changes induced in 'stacked cup' carbon nanofibers by heat treatment," Carbon, vol. 41, no. 10, pp. 1941-1947, 2003.

[25] R. Andrews, D. Jacques, D. Qian, and E. C. Dickey, "Purification and structural annealing of multiwalled carbon nanotubes at graphitization temperatures," Carbon, vol. 39, no. 11, pp. 16811687, 2001.

[26] A. Cuesta, P. Dhamelincourt, J. Laureyns, A. Martínez-Alonso, and J. M. D. Tascón, "Raman microprobe studies on carbon materials," Carbon, vol. 32, no. 8, pp. 1523-1532, 1994.

[27] H. Seuk Youn, H. Ryu, T.-H. Cho, and W.-K. Choi, "Purity enhancement and electrochemical hydrogen storage property of carbon nanofibers grown at low temperature," International Journal of Hydrogen Energy, vol. 27, no. 9, pp. 937-940, 2002.

[28] R. Guzmán, Optimización de propiedades mecánicas y eléctricas de nanofibras de carbono/epoxi [Ph.D. thesis], University of Zaragoza, Zaragoza, Spain, 2007.

[29] P. V. Lakshminarayanan, H. Toghiani, and C. U. Pittman Jr., "Nitric acid oxidation of vapor grown carbon nanofibers," Carbon, vol. 42, no. 12-13, pp. 2433-2442, 2004. 

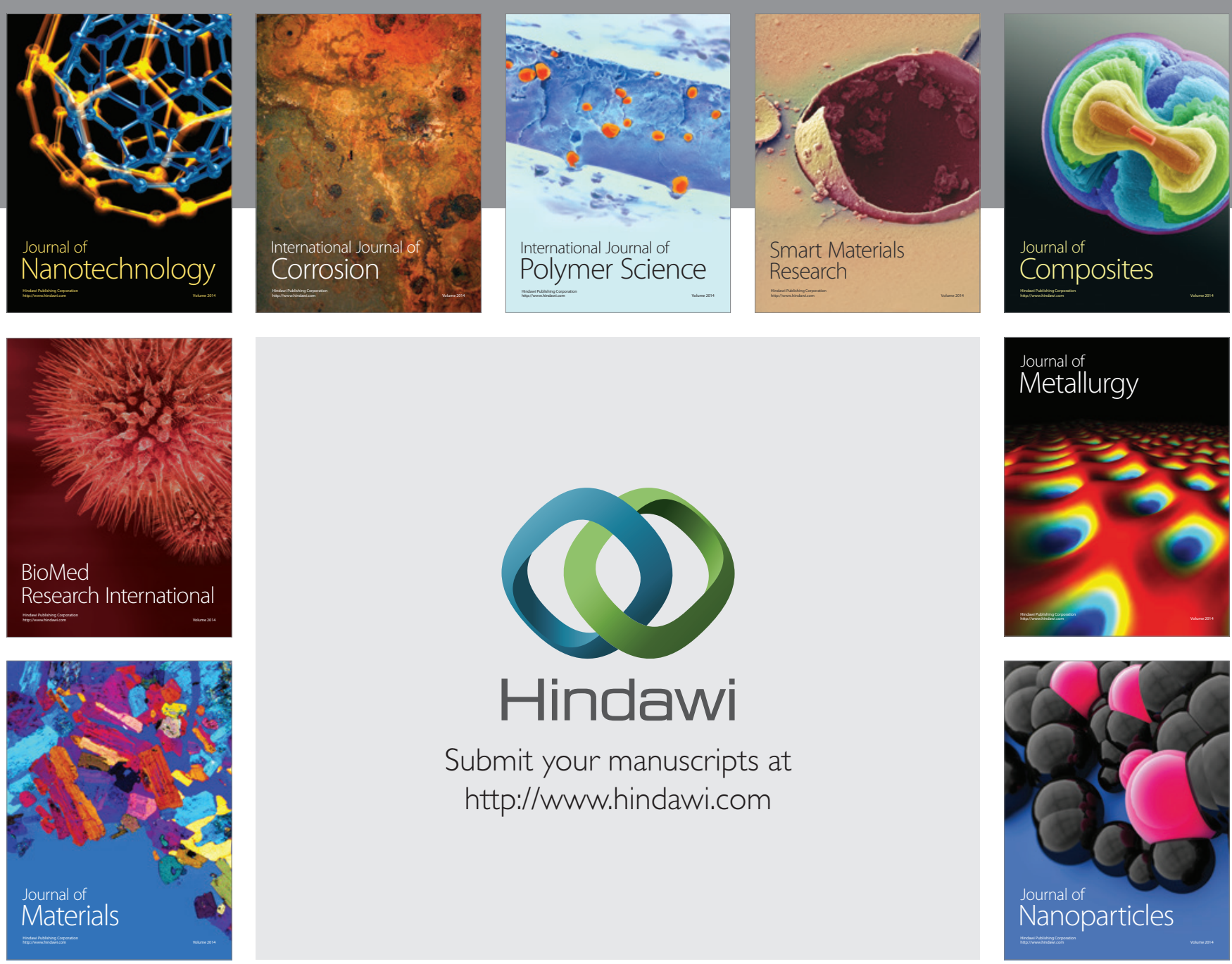

Submit your manuscripts at http://www.hindawi.com
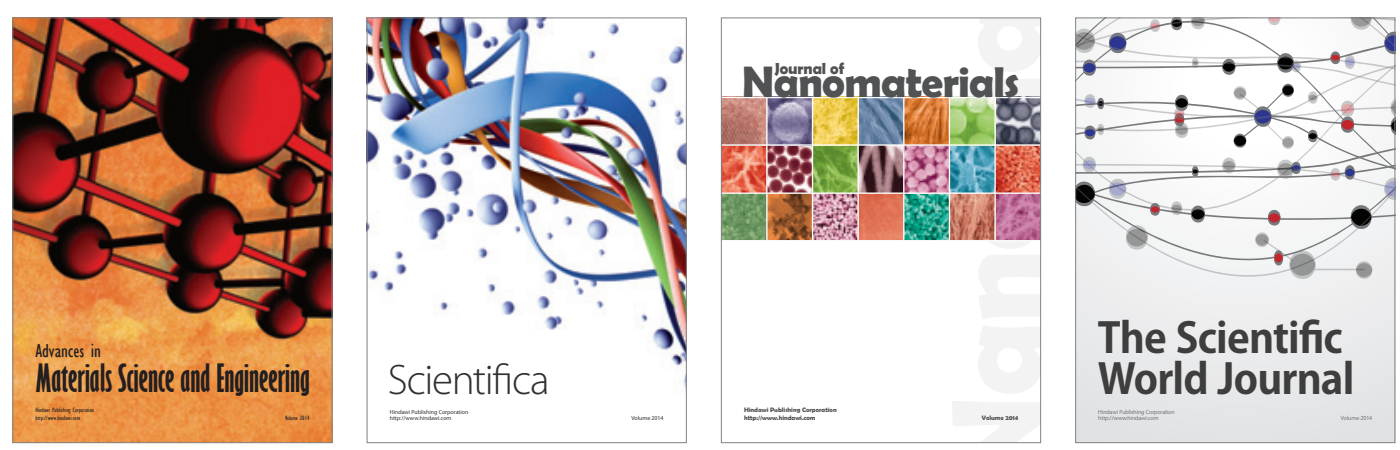

\section{The Scientific World Journal}
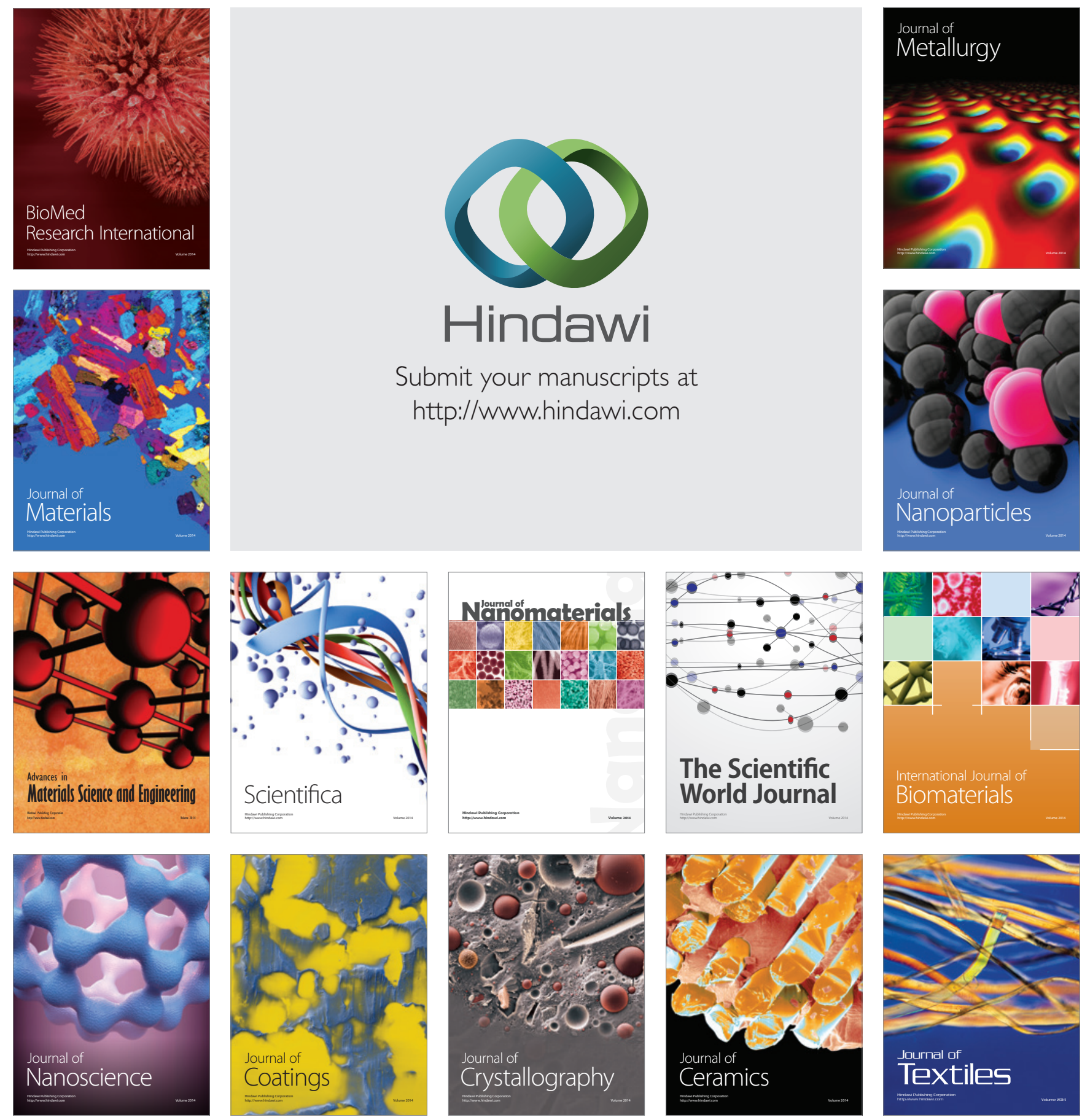